\title{
Bilateral Activity-Dependent Interactions in the Developing Corticospinal System
}

\author{
Kathleen M. Friel ${ }^{1,3}$ and John H. Martin ${ }^{1,2,3}$ \\ Departments of ${ }^{1}$ Neuroscience and ${ }^{2}$ Neurological Surgery and Psychiatry, Columbia University, New York, New York 10032, and ${ }^{3}$ New York State \\ Psychiatric Institute, New York, New York 10032
}

Activity-dependent competition between the corticospinal (CS) systems in each hemisphere drives postnatal development of motor skills and stable CS tract connections with contralateral spinal motor circuits. Unilateral restriction of motor cortex (M1) activity during an early postnatal critical period impairs contralateral visually guided movements later in development and in maturity. Silenced M1 develops aberrant connections with the contralateral spinal cord whereas the initially active M1, in the other hemisphere, develops bilateral connections. In this study, we determined whether the aberrant pattern of CS tract terminations and motor impairments produced by early postnatal M1 activity restriction could be abrogated by reducing activity-dependent synaptic competition from the initially active M1 later in development. We first inactivated M1 unilaterally between postnatal weeks 5-7. We next inactivated M1 on the other side from weeks 7-11 (alternate inactivation), to reduce the competitive advantage that this side may have over the initially inactivated side. Alternate inactivation redirected aberrant contralateral CS tract terminations from the initially silenced M1 to their normal spinal territories and reduced the density of aberrant ipsilateral terminations from the initially active side. Normal movement endpoint control during visually guided locomotion was fully restored. This reorganization of CS terminals reveals an unsuspected late plasticity after the critical period for establishing the pattern of CS terminations in the spinal cord. Our findings show that robust bilateral interactions between the developing CS systems on each side are important for achieving balance between contralateral and ipsilateral CS tract connections and visuomotor control.

Key words: voluntary movement; locomotion; visual guidance; spinal cord; motor cortex; activity dependence; cerebral palsy

\section{Introduction}

The corticospinal (CS) system is essential for skilled movements in many animals and in humans. Development of the CS system in cats depends on motor cortex (M1) activity and limb use during an early postnatal critical period (Martin et al., 1999; Friel and Martin, 2005; Martin, 2005). This is when activity-dependent competition between the developing CS systems from each hemisphere guides pruning of exuberant ipsilateral projections and increases axon terminal density. This process leads to establishing the predominantly contralateral pattern of CS tract connections with spinal motor circuits. In humans, the CS system also is refined postnatally from a bilateral to a predominantly contralateral organization (Eyre et al., 2007).

Restricting M1 activity in one hemisphere during this critical period, between postnatal week 5 (PW5) and PW7, shifts the balance of activity-dependent competition to the active side and permanently alters development of the CS systems. Silenced CS

\footnotetext{
Received June 20, 2007; revised July 25, 2007; accepted Aug. 28, 2007.

This work was supported by National Institutes of Health Grant NS33835. We acknowledge the assistance of Xiu Li Wu for histochemistry and immunohistochemistry, Dan Raz for developing the imaging software, Germa Asfaw and Dr. M. Osman for veterinary care, and Erin Nunnink, Cedrick Mendoza-Tolentino, Dr. Iran Salimi, and Bernice Sist for help with training and analysis. We thank Dr. Jason Carmel for helpful comments on a previous version of this manuscript.

Correspondence should be addressed to Dr. John H. Martin, Department of Neuroscience, Columbia University, 1051 Riverside Drive, New York, NY 10032. E-mail: jm17@columbia.edu.

DOI:10.1523/JNEUROSCI.2814-07.2007

Copyright $\odot 2007$ Society for Neuroscience $\quad$ 0270-6474/07/2711083-08\$15.00/0
}

axons fail to develop strong contralateral connections within appropriate motor laminas of the spinal cord, but rather terminate dorsally in mechanosensory laminas. The active CS system of the other hemisphere maintains bilateral projections into maturity. Cats with these aberrant CS tract terminations have permanent contralateral endpoint control deficits (Martin et al., 2000; Friel et al., 2007). Using transcranial magnetic stimulation in children with hemiplegic cerebral palsy (CP), a sparse contralateral CS projection from the affected cortex and a bilateral projection from the unaffected cortex is seen (Eyre et al., 2001). These findings suggest that developing CS axon terminations from M1 in each hemisphere interact on common spinal motor circuits to secure stable contacts. Balanced competition between the two sides is essential for normal connectivity and motor function (Martin and Lee, 1999).

After PW5-PW7, CS axon terminations in cats become stronger (Meng and Martin, 2003; Meng et al., 2004) and the M1 motor representation develops (Chakrabarty and Martin, 2000). This is also when the animals' repertoire of skilled movements expands (Martin and Bateson, 1985). What is the role of activitydependent competition between the developing CS systems when the projections are predominantly contralateral? Importantly, can activity-dependent competition be harnessed to restore function after early activity imbalance leaves the system with aberrant connections? To answer these questions, in the present study we examined activity-dependent competition between the CS sys- 
tems later in development. We inactivated M1 unilaterally between PW5 and PW7 and then inactivated M1 on the other side between PW7 and PW11, as CS terminations get stronger. This second, or alternate, inactivation reduces the competitive advantage that this side may have over the initially inactivated side.

We found that activity-dependent competition between the developing CS systems is important later in development, as the system matures connection strength and function. Alternate inactivation restored the normal pattern of CS terminations in the spinal cord and contralateral visually guided motor skills. The developing functions of the CS tract on one side of the spinal cord reflect the balanced contributions from M1 on each side. Our findings also provide insights into mechanisms underlying development of aberrant CS connectivity and function in hemiplegic CP.

\section{Materials and Methods}

Animals were obtained from an Association for Assessment and Accreditation of Laboratory Animal Care-accredited supplier. Kittens were delivered in litters of four or five along with a lactating mother at postnatal week 4. Columbia University and the New York State Psychiatric Institute Institutional Animal Care and Use Committees approved all experimental procedures.

General surgical procedures. For all surgical procedures, animals were administered atropine $(0.04 \mathrm{mg} / \mathrm{kg}$ i.m. $)$. A mixture of acepromazine $(0.03 \mathrm{mg} / \mathrm{kg}$ i.m.) and ketamine hydrochloride $(32 \mathrm{mg} / \mathrm{kg}$, i.m.) was given to induce anesthesia. Cats were intubated after anesthesia was induced and were maintained in an areflexive condition during surgery using $1-2 \%$ isofluorane. Animals were given a broad-spectrum antibiotic at the time of surgery (cephalozine; $25 \mathrm{mg} / \mathrm{kg}$, i.m.) and an analgesic after surgery (burprenorphine; $0.03 \mathrm{mg} / \mathrm{kg}$, i.m.). Animals resumed nursing after recovery from anesthesia, and were given supplemental milk [feline milk replacement (KMR); PetAg, Hampshire, IL] as needed to ensure adequate weight gain.

M1 activity blockade. To block neuronal activity, the $\mathrm{GABA}_{\mathrm{A}}$ agonist muscimol (10 mm in sterile saline; Sigma, St. Louis, MO) was continuously infused using an osmotic minipump (model 2002; Alzet, Cupertino, CA) $(0.5 \mu \mathrm{l} / \mathrm{hr})$ into the center of the motor cortex forelimb representation, located primarily in the lateral sigmoid gyrus (Chakrabarty and Martin, 2000), as in our previous studies (Martin et al., 1999; Friel and Martin, 2005). A 28-guage hypodermic needle cannula (Alzet), beveled at the tip, was connected with vinyl tubing (size 4; Scientific Commodities, Lake Havasu City, AZ) to the pump. The cannula was inserted below the pial surface. The cannula was fixed to the skull with screws and dental acrylic cement. Neuronal activity was blocked in left M1 from PW5 to PW7, and then in right M1 from PW7 to PW11. The osmotic pump delivered muscimol for 2 weeks. During the inactivation of right M1 from PW7 to PW11, it was necessary to replace the pump at PW9. The cannula and pump were removed at the end of the infusion period.

We have shown using the metabolic marker cytochrome oxidase that this infusion maximally inhibits a $2.5-3 \mathrm{~mm}$ patch of cortex at the infusion site and less inhibition for an additional $4-5 \mathrm{~mm}$ (Martin et al., 1999). This inactivation also produces a reduction in the level of neuropil immunostaining of the calcium binding protein parvalbumin over approximately the same distance as observed for the cytochrome oxidase reduction (Friel et al., 2007). In previous studies, we verified that the infusion did not produce a lesion by comparing, within the infused and noninfused cortex, cell body density, the distribution of neurofilament-F (SMI-32) immunoreactivity, and parvalbumin cell body staining. We also found that the medullary pyramid cross-sectional areas on each side and SMI-32 staining in the lateral spinal column on each side were not different (Martin et al., 1999, 2000; Friel et al., 2007).

Tracer injections. Biotinylated dextran anime (BDA; 5\% in PBS; Invitrogen, Eugene, OR) and Lucifer yellow dextran (LY; 1\% in PBS; Invitrogen) were pressure-injected into M1 4 weeks before killing the animal to examine the CS projection from neurons in the forelimb areas of each hemisphere. BDA was injected into the left (first inactivated) M1 in most animals, whereas LY was injected into the left cortex in one animal. The reverse was done for the right (second inactivated) M1. Injections were made at least $2 \mathrm{~d}$ after cessation of muscimol infusion. In the alternate inactivation group, injections in two cats were made at least $2 \mathrm{~d}$ after the first inactivation ended, whereas in four cats, injections were made at least $2 \mathrm{~d}$ after cessation of the second inactivation. Previous experiments indicated that tracer injected at the time of an intracortical infusion failed to label CS terminals. This was likely caused by tracer washout because the injection sites were only weakly stained for BDA. Uptake and anterograde transport of BDA by $\mathrm{M} 1$ are not dependent on local neural activity (Friel and Martin, 2005).

All injections were made under visual guidance within a band of cortex just lateral to the tip of the cruciate sulcus, as in our previous studies ( $\mathrm{Li}$ and Martin, 2001; Friel and Martin, 2005). These injections were all within the primary motor cortex. Three injections of BDA, $300 \mathrm{nl}$ each, were made in each cat. The injections were separated by $1.5 \mathrm{~mm}$ and placed rostral, lateral, and posterior to the former cannula implantation site, which was just lateral to the tip of the cruciate sulcus. We insured that differences in the distribution of CS axons in the spinal cord were not attributable to differences in the locations of injections sites. In all animal groups, the locations of injection sites, relative to the cruciate sulcus, were identical. Importantly, after the initial inactivation, animals were randomly assigned either to receive the alternate inactivation or have no additional treatment. Injections were made $1.5 \mathrm{~mm}$ below the pial surface.

Histology and tracer histochemistry. Four weeks after tracer injection, cats were deeply anesthetized with sodium pentobarbital (30 mg/kg, i.v.) and perfused transcardially with warm saline, followed by a solution of $4 \%$ paraformaldehyde, $\mathrm{pH}$ 7.4. Heparin was injected (200-500 U, i.v.) at the onset of perfusion. For perfusion, a peristaltic pump was used at a predetermined flow rate that depended on the animal's weight. The total perfusion time was 20-30 min. The brain and spinal cord were removed, postfixed in the same fixative at $4^{\circ} \mathrm{C}$ for $2-3 \mathrm{~h}$, and then transferred to $20 \%$ sucrose in $0.1 \mathrm{~m}$ phosphate buffer overnight.

Frozen transverse sections $(40 \mu \mathrm{m})$ through the cervical spinal cord (C7-C8) were cut and processed for BDA histochemistry and LY immunohistochemistry to determine the distribution of labeled CS terminals. Parasagittal sections through the cortex were cut and alternate sections were processed for BDA or LY, to determine the location of tracer injection sites, and Nissl-stained for assessing cortical cytoarchitecture. For visualization of $\mathrm{BDA}$, sections were incubated in PBS containing $1 \%$ avidin-biotin complex reagent (ABC kit; Vector Laboratories, Burlingame, $\mathrm{CA}$ ) and $0.2 \%$ Triton for $2 \mathrm{~h}$ at room temperature. After rinsing, sections were incubated with the chromogen diaminobenzidine (DAB; Sigma) for 6-30 min. After rinsing, sections were mounted on gelatincoated slides, air-dried overnight, dehydrated, and coverslipped. For visualization of LY, sections were incubated at $4^{\circ}$ overnight in PBS containing $0.01 \%$ rabbit anti-LY antibody (Invitrogen) in blocking buffer (3\% goat serum in $1 \times \mathrm{PBS}$ with $0.2 \%$ Tween, $\mathrm{pH} 7.4$ ). After rinsing, sections were incubated for $2 \mathrm{~h}$ at room temperature in blocking buffer containing $0.2 \%$ anti-rabbit antibody conjugated to peroxidase, $\mathrm{pH}$ 7.4. After rinsing, sections were incubated with the chromogen DAB for 5-30 min. Sections were mounted on gelatin-coated slides, air-dried overnight, dehydrated, and coverslipped.

Quantitative analysis of the topography of CS terminations. We developed a quantitative method for determining the topographic distribution of label within the gray matter in the cervical enlargement. For this analysis, BDA- and LY-labeled axons were traced from transverse spinal sections at $200 \times$ magnification using Neurolucida (Microbrightfield, Williston, VT), by moving the cursor along the length of the labeled axon. Contralateral and ipsilateral projections were traced. CS terminations are defined as axon label within the gray matter in spinal sections. This labeling was present as either preterminal or terminal, which we do not distinguish. Boutons are defined as punctate axonal swellings (i.e., varicosities) with a diameter more than three times the diameter of the nonvaricose axon. Bouton sites along axons were marked. Boutons are putative presynaptic sites. We showed previously that such profiles colocalize synaptophysin (Meng et al., 2004). Axon label comprising CS terminations was traced and boutons were marked in Neurolucida as separate 
Control

A Axons

A1.

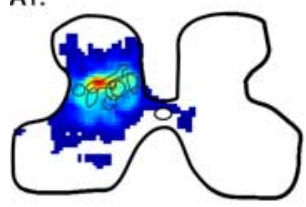

B Varicosities

B1.

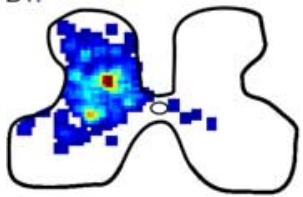

Unilateral inactivation
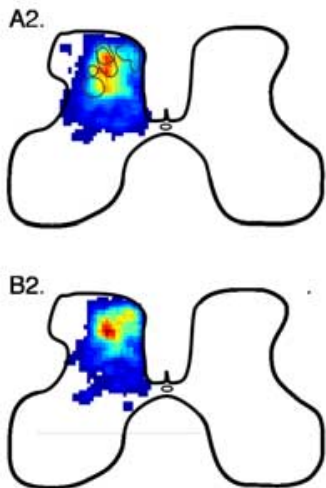

Alternate inactivation
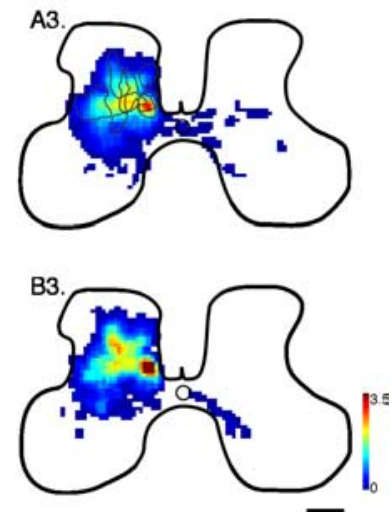

Figure 1. Alternate inactivation promotes redistribution of $C S$ terminations to intermediate and ventral motor laminas after previous M1 inactivation. Color-coded average density maps are shown for age-matched controls (left column; $n=4$ ), animals with unilateral inactivation (center; $n=4$ ), and animals with alternate inactivation (right; $n=6$ ). Axon density (terminals and preterminal axons) maps for are shown in $\boldsymbol{A}$ and bouton density maps in $\boldsymbol{B}$. The contours in $\boldsymbol{A}$ graph the densest regions (yellow; maximal $40 \%$ ) from the individual animals comprising the averages. Scale bar, $500 \mu \mathrm{m}$; color scale: $\boldsymbol{A}, 0-216 \mu \mathrm{m}$ axon/ $\mu \mathrm{m}^{2}$ area; $\boldsymbol{B}, 0-3.5 \times 10^{-2}$ boutons/ $\mu \mathrm{m}$.

structures and analyzed separately. For each label used in each cat (BDA or LY), all axon label was traced on each section. The number of sections traced was determined by the amount of label on each section. Sections were added until the total length of axon that had been traced reached 25,000 $\mu \mathrm{m}$.

Tracings of axon label and boutons were separately exported and quantified using a suite of programs written in the Matlab (MathWorks, Natick, MA) programming environment. Briefly, a program divided the gray matter into $40 \times 40 \mu \mathrm{m}$ square regions of interest (ROI). For each ROI, we computed the mean density of traced axons or marked boutons. All axonal tracings were one pixel thick; boutons were one pixel. This corresponds to the amount of label (i.e., total number of pixels) in the ROI divided by the area of the ROI. A matrix of mean axon or bouton density was generated in Matlab that preserved the mediolateral and dorsoventral dimensions of the distribution of label in the gray matter. At the same time, the borders of the gray matter were traced to obtain a precise correspondence between labeling and spinal cord structure. We quantified the regional axon and bouton density to generate maps of regional distribution of axonal label distribution and to quantify the amounts of label in different spinal laminas. Density is represented according to a color scale, from the lowest density (blue) to the highest (red). Regional distribution maps were generated for individual animals and averaged maps for all animals within a particular treatment group. For the averages, we aligned the data from different animals according to the point of intersection between the gray matter above the central canal and the dorsal median septum.

To compare the locations of label across animals, we transformed the regional distribution maps (i.e., dorsoventral and mediolateral) to dorsoventral distributions only. This was done by summing all label present at a single depth. Because the size of the gray matter differs from animal to animal, we normalized dorsoventral distributions of labeling. For this analysis, we marked the dorsal and ventral gray matter borders and interpolated 1000 rows between. We grouped labeling according to Rexed's laminas: 2-4 and 5-7.

Behavioral testing on horizontal ladder-walking task. We examined performance of animals subjected to the alternate inactivation while walking on a horizontal ladder $(88 \mathrm{~cm}$ long, $18.4 \mathrm{~cm}$ wide; $0.9 \mathrm{~cm}$ square rungs; 6 $\mathrm{cm}$ rung interval) (Friel et al., 2007). Training and testing occurred between PW10 and PW16. Cats were placed on a platform at one end of the ladder and meat cubes were placed at the other end. Testing was videotaped. During testing, the cat walked across the ladder from the start platform to the food reward. After the cat traversed the ladder in one direction, the food reward for the next trial was placed on the other end of the ladder, requiring the cat to traverse the ladder in both directions. This ensured that both sides of the cat were captured on film. To prevent cats from memorizing rung position, we placed them at different positions on the platform for each trial while keeping the distance between rungs constant. This resulted in their starting to step on the rungs with either forelimb. Moreover, the first ladder rung that was stepped on differed from trial-to-trial. We previously showed that control animals adjusted their step distance in accurate proportion to surprise changes in rung distance (Friel et al., 2007).

Analysis of ladder step movements. Videotapes of testing sessions were imported into a video editing program [iMovie, for the Apple (Cupertino, CA) Macintosh computer]. Images from the video files were analyzed at $30 \mathrm{~Hz}$, pausing at the frame in which the paw made contact with the rung. We measured the distance that the tip of the cat's forepaw extended in front of the rung of the ladder (termed forward distance). The forward distance was measured on a flat computer screen. Distance measures from the computer screen were converted to centimeters by scaling according to a calibrated distance on each video file. We compiled a database of mean forward distance of animals after alternate inactivation. These data were compared with control data from a previous study (Friel et al., 2007): age-matched noninactivated animals, M1 saline infusion, and unilateral inactivation.

\section{Results}

In this study, we first inactivated the forelimb area of left M1 between PW5 and PW7, which is the critical period for establishing the pattern of CS axon termination in the spinal cord (Theriault and Tatton, 1989; Alisky et al., 1992; Li and Martin, 2001; Li and Martin, 2002). We then inactivated the right M1 forelimb area between PW7 and PW11 (termed alternate inactivation; $n=$ 6 cats). This is the period when CS axon terminals develop stronger connections with spinal motor circuits (Meng and Martin, 2003; Meng et al., 2004) and when the M1 motor representation forms (Chakrabarty and Martin, 2000). Cats were perfused at the cessation of the second inactivation (PW $11 ; n=2$ ) to examine the effects of alternate inactivation on CS tract axon terminations in the cervical enlargement or 1 month later (PW 15 or $16 ; n=4$ ) to examine the effects of alternate inactivation on motor skills from PW10 to PW15/16 and regional CS terminations and bouton density. Morphological and behavioral data from the alternate inactivated animals were compared with data from unilaterally inactivated cats $(n=4)$ and age-matched, noninactivated controls $(n=4)$. In all cats, anterograde tracers were injected into M1 4 weeks before perfusion.

\section{Redistribution of corticospinal terminations and boutons after alternate inactivation}

The topographic distribution of labeled CS axon terminations and boutons originating from left M1 was examined in transverse spinal sections at the $\mathrm{C} 8$ level in each animal group (Fig. 1). Tracings of axon labeling were converted to a regional distribution map and represented as a color scale (Fig. $1 A$ ). CS terminations in age-matched controls (noninactivated) cats were, on average, densest within the deepest layer of the dorsal horn (lamina 5) and the intermediate zone (laminas 6 and 7) (Fig. 1A1), similar to previous findings (Theriault and Tatton, 1989; Alisky et al., 
1992; Friel and Martin, 2005). Sparse ipsilateral terminations were present in the medial portion of lamina 7. The contours mark the locations of regions containing high densities of terminations ( $\geq 40 \%$; corresponding approximately to yellow) in the individual animals comprising the average. All of the maximal density zones in the control animals (Fig. 1 A1) are within laminas 5-7. Unilateral inactivation shifted the densest zone of CS terminations to more dorsal and lateral territories (laminas 2-4) (Fig. 1A2). The present animals, together with our previous data for PW 15/16 animals (Friel and Martin, 2005), clearly show that, after inactivation between PW5 and PW7, CS axon terminations are densest in a region that is more dorsal and lateral to that of controls. Ipsilateral terminations were absent after unilateral inactivation. In contrast, after alternate inactivation, CS axon terminal distribution (Fig. 1A3) returned to a pattern similar to that of age-matched controls, with maximal density in the deepest layer of the dorsal horn and the intermediate zone (laminas 5, 6, and 7). Sparse ipsilateral terminations were present in the medial portion of lamina 7 after the alternate inactivation, similar to the controls.

Not surprisingly, the regional distribution of synaptic boutons closely matched the distribution of axons (Fig. $1 B$ ). In agematched controls, synaptic bouton density was greatest within laminas 5-7 (Fig. 1 B1). Unilateral inactivation shifted the densest zone of boutons dorsally to laminas $2-4$ (Fig. 1 B2). In contrast, after alternate inactivation (Fig. 1 B3), bouton distribution was similar to that of age-matched controls. Reconstructions of individual CS tract terminal and preterminal branches after alternate inactivation also showed a redistribution to more ventral laminas (supplemental Fig. 1, available at www.jneurosci.org as supplemental material). Thus, alternate inactivation produced a redistribution of CS axon terminals and presynaptic boutons from an aberrant location for M1 terminations in the dorsolateral portions of the dorsal horn to a normal location in the deep dorsal horn and intermediate zone.

It should be noted that the redistribution of CS axon terminations and boutons after alternate inactivation is not caused by differences in the locations of tracer injections for the different animal groups. As indicated in Materials and Methods, all animals received the same number of injection into the same three sites lateral to the tip of the cruciate sulcus. This is the forelimb representation of $\mathrm{M} 1$, in both controls and after unilateral inactivation (Chakrabarty and Martin, 2000; Chakrabarty et al., 2007). Animals were randomly chosen to receive the alternate inactivation or no additional treatment.

To quantify the shift in the laminar distribution of CS axon terminations and boutons, we plotted the normalized dorsoventral distribution of axon labeling and bouton label in the gray matter (Fig. 2) (see Materials and Methods). Averaged ( \pm SEM) dorsoventral plots show that unilateral inactivation shifted CS terminations and boutons to more dorsal laminas. After alternate inactivation, CS axon termination and bouton distribution returned to a pattern similar to age-matched controls, with densities concentrated in the deep dorsal horn and intermediate zone. To determine whether the differences in the dorsoventral distribution of CS axon terminations were significant, we compared the total amount of labeling within the superficial laminas (2-4) (Fig. 3A) and the deep (5-7dorsal) laminas (Fig. 3B). Importantly, the laminar distribution of CS terminations after alternate inactivation was not significantly different from controls. Moreover, there was significantly less dorsal labeling after alternate inactivation compared with unilateral inactivation (Fig. 3A) and significantly more ventral labeling (Fig. 3B) (laminas 2-4,

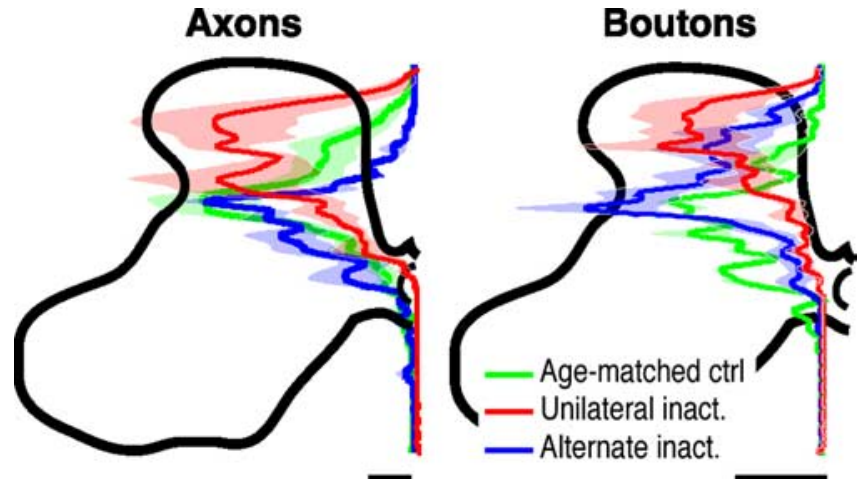

Figure 2. Unilateral inactivation (inact.) shifts the distribution of CS terminations dorsally in the gray matter and alternate inactivation restores terminations to the intermediate and ventral laminas. Dorsoventral distributions of axon (left) and bouton (right) density from the dorsal to ventral margins of the contralateral gray mater are shown. Solid lines plot the mean pixel values at each normalized depth; light shading plots \pm SEM. Data from controls (ctrl.) are shown in green, unilateral inactivation in red, and alternate inactivation in blue. To normalize for differences in the size of the gray matter across animals, all graphs were interpolated to 1000 points between the dorsal and ventral margins of the gray matter. Scale bars: axons, $500 \mu \mathrm{m}$ axon $/ \mu \mathrm{m}^{2}$ area; varicosities, $5 \times 10^{-3}$ boutons $/ \mu \mathrm{m}$.
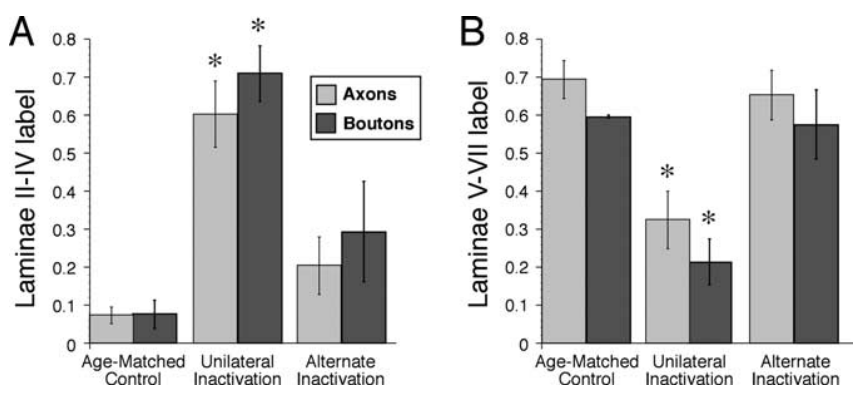

Figure 3. $\boldsymbol{A}, \boldsymbol{B}$, The proportion of axonal labeling (light gray) and boutons (dark gray) within laminas 2-4 (A) and laminas 5-7 (B) after alternate inactivation are similar to controls. Data from controls, unilateral inactivation, and alternate inactivation are shown. Asterisks show significant differences for axon labeling and bouton density between the unilateral inactivation group and either the controls $(p<0.01)$ or the alternate inactivation $(p<0.01)$ groups. Error bars indicate SEM.

ANOVA, $F=5.87, p=0.019$; laminas 5-7, ANOVA, $F=9.99$, $p=0.003)$. There was a significant difference in label density in laminas 2-4 and laminas 5-7 between groups. After unilateral inactivation, there was significantly more label in dorsal laminas (Fig. 3A) (Bonferroni's/Dunn's test, $p=0.007$ ) and significantly less label in ventral laminas (Fig. 3B) (Bonferroni's/Dunn's test, $p=0.002)$ than in age-matched noninactivated controls. We also examined the differences in the dorsoventral distribution of CS boutons. The laminar distribution of boutons after alternate inactivation was not significantly different from controls. Like axon density, there were significantly fewer boutons in the superficial laminas after unilateral inactivation (Fig. $3 A$ ) and significantly more ventrally (Fig. $3 B$ ) compared with controls (laminas 2-4, ANOVA, $F=5.02, p=0.344$; laminas $5-7$, ANOVA, $F=5.48$, $p=0.028)$.

We next compared the effects of alternate inactivation at 11 weeks $(n=2)$, which is immediately after the alternate inactivation, and at 15 weeks $(n=4)$, to permit behavioral testing (see below). Interestingly, the dorsoventral distributions of axon and bouton label density were the same in the two age groups (Fig. 4). There were no significant differences in the amount of axonal label or boutons in laminas 2-4 (ANOVA, axons, $F=6.52, p=$ 0.063 ; boutons, $F=2.95, p=0.16$ ) or laminas 5-7 (ANOVA, 


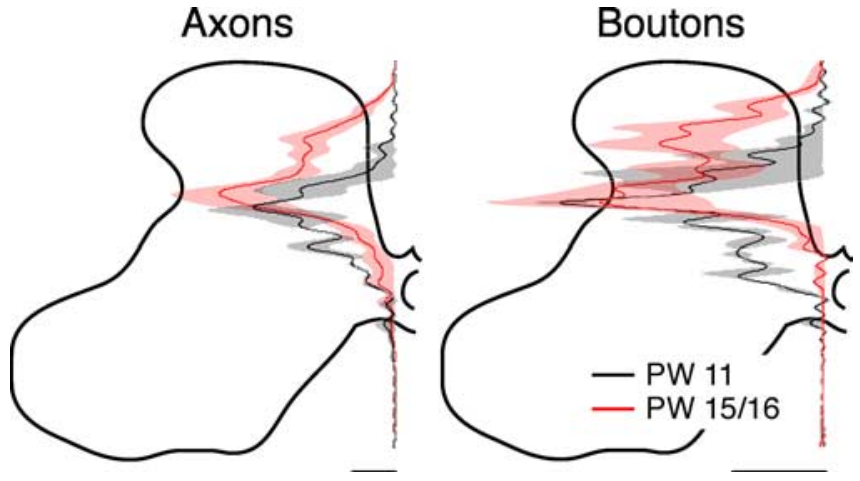

Figure 4. CS terminations have similar distribution immediately after the alternate inactivation (PW 11) and 4 weeks later (PW 15). Dorsoventral distributions of axon (left) and bouton (right) density from the dorsal to ventral margins of the contralateral gray mater are shown for animals examined at PW 11 ( $n=2$; black) and PW 15/16 ( $n=4$; red). Solid lines and shading are similar to Figure 2 . Scale bars: axons, $500 \mu \mathrm{m}$ axon $/ \mu \mathrm{m}^{2}$ area; varicosities, $5 \times 10^{-3}$ boutons/ $\mu \mathrm{m}$.

axons $F=0.66, p=0.46$; boutons, $F=1.34, p=0.31$ ). This shows that the effects of the alternate inactivation on axon distribution and bouton density occurred during the second inactivation period, not during the postinactivation survival period.

\section{Alternate inactivation reduces aberrant ipsilateral terminations}

The initially active side maintains "transient" ipsilateral terminations that could be maladaptive because they might out-compete the silenced contralateral projection for termination space. We next determined whether the alternate inactivation reduced the density of aberrant ipsilateral terminations from the initially active (right) M1. As indicated previously, ipsilateral terminations are normally sparse, as shown in Figure $5 A$ (data replotted from Fig. $1 A$ ). Bouton density had a similar distribution as axon label (data not shown). After unilateral inactivation $(n=3)$ (Fig. $5 B)$, there were abundant decussating axons in lamina 10, indicating that the ipsilateral terminations are largely caused by axons crossing a second time at the segmental level. Presumably, these are maintained ipsilateral terminations that are normally eliminated by PW7. The density was highest in medial lamina 7, with sparser terminations extending from this zone into laminas 5, 6, and 8 . Overall, however, the density was much lower than for contralateral terminations. A similar pattern of boutons was present (data not shown).

After the alternate inactivation $(n=3)$ (Fig. $5 C)$, the extent of ipsilateral labeling was less than after unilateral inactivation alone. Contours of averaged contralateral axon terminations from the initially inactivated side are superimposed on the regional distribution maps [inner contour, region of densest label, corresponding to yellow region (and above) in Fig. 1A; outer contour, region of most labeling, corresponding to light blue region (and above) in Figure $1 A]$. Without the alternate inactivation, the silenced side projections are excluded entirely from the territory of the aberrant ipsilateral terminations. In contrast, with the alternate inactivation, some overlap developed. As a percentage of the total area of the ipsilateral gray matter, ipsilateral labeling was significantly less after alternate inactivation $(4.6 \pm 0.6 \%$ after alternate inactivation compared with $16.5 \pm$ $7.1 \%$ after unilateral inactivation; Mann-Whitney $U$ test, $z=$ $1.96, p=0.05)$. The reduction in ipsilateral CS terminations was not caused by a muscimol-related drug effect because the distribution of contralateral labeling was not significantly reduced
(Mann-Whitney $U$ test, $z=0.21, p=0.83$ ). After alternate inactivation, the ipsilateral terminations are less able to maintain connectivity. Considering this finding together with the anatomical data presented above, after the alternate inactivation, the initially silenced axons may be more competitive in securing termination space.

\section{Recovery of visuomotor skill after alternate inactivation}

After unilateral inactivation, visuomotor control using the contralateral forelimb is permanently impaired, never recovering spontaneously (Martin et al., 2000; Friel et al., 2007). Our previous study showed, for visually guided locomotion, that control animals place each paw squarely and securely on each ladder rung (Friel et al., 2007). After unilateral M1 inactivation, the paw contralateral to the previous inactivation was placed forward to, and often slipped in front of, the ladder rungs. That study tested performance up to 21 weeks after the unilateral inactivation without significant improvement, likely indicating a permanent impairment. The paw ipsilateral to the inactivation was placed on the rungs similar to control cats. In the present study, we determined whether the alternate inactivation promoted recovery of motor control function ( $n=4$ cats). We measured the forward distance for each step for the paw contralateral to the initial inactivation. Forward distance after alternate inactivation was compared with our published data for age-matched controls and animals with unilateral inactivation (Friel et al., 2007). The paw contralateral to the alternate inactivation showed a transient overstepping to the blockade (supplemental Fig. 2, available at www.jneurosci. org as supplemental material).

We examined two features of performance in animals receiving alternate inactivation: (1) recovery time (when the animal's forward distance became statistically indistinguishable from noninactivated controls) and (2) the final forward distance value. For recovery time, we acclimatized all animals to ladder walking at weekly intervals during the alternate inactivation. Then we tested performance during the last few days of the alternate inactivation to determine whether performance had recovered. Mean forward distance during the last week of alternate inactivation was $2.98 \pm 0.11 \mathrm{~cm}$. This was not different from animals receiving unilateral inactivation $(t=1.69 ; p=0.14)$ (Friel et al., 2007). After animals recovered from the surgery to remove the infusion cannula, we re-tested performance on the ladder twice-weekly for each animal.

Figure $6 \mathrm{~A}$ shows the change in forward distance for representative animals after unilateral and alternate inactivation. Distances for the forepaw contralateral to the first inactivation are plotted as \pm SEM. Average forward distance for control cats is plotted as a dotted line $(n=4)$. Forward distance was greater than controls for $>2$ months after unilateral inactivation, strongly suggesting the deficit is permanent. After alternate inactivation, forward distance returned to control levels by 1 month after the second inactivation. The percentage change in step distance (forward distance at the time of recovery or perfusion divided by the forward distance at PW10) for all animals examined (Fig. 6B) shows that, as a group, the alternate inactivation animals recovered between PW11.4 and PW14.3 (average 12.9 \pm 0.54 weeks). ANOVA revealed a significant effect of the alternate inactivation on absolute forward distance (ANOVA, $F=35.558, p<0.0001$ ). In contrast, forward distance of animals in the unilateral inactivation group never returned to control levels. Three animals got worse, whereas one improved slightly but never recovered to a control forward distance. The absolute forward distance of the forepaw contralateral to the first inactivation (PW5-PW7) for all 
cats in the alternate inactivation group at 15 weeks was $1.91 \pm 0.08$, statistically indistinguishable from controls (1.71 for controls; Fisher's protected least significant difference, $p=0.16$ ). Thus, unilateral inactivation without additional intervention produced permanent contralateral motor deficits, whereas alternate inactivation promoted recovery of overstepping in the paw contralateral to the first inactivation.

\section{Discussion}

Balanced activity-dependent competition between the CS systems in each hemisphere during an early postnatal critical period is necessary for the development of stable connections with spinal motor circuits and for skilled motor control. When M1 activity is restricted on one side during this critical period, visually guided movements become impaired contralaterally and aberrant CS tract connections develop bilaterally (Martin, 2005). Although these changes persist into maturity without additional manipulations, they are not immutable. Harnessing activity-dependent competition later in development restores connectivity and function, revealing an unsuspected late plasticity. Interestingly, the motor systems required time to learn to use the new connections because visually guided control did not fully recovery until several weeks after restoration of connectivity. This interplay between the developing CS systems on each side is important for establishing the laterality of CS tract connections and normal visuomotor control.

Capacity for topographic reorganization after the critical period

Topographic refinement of the CS system occurs during the first two postnatal months in the cat (Theriault and Tatton, 1989; Alisky et al., 1992; Li and Martin, 2001; Li and Martin, 2002). A growth-associated protein that marks this period in the cat (microtubule associated protein 1B; similar to GAP 43) (Schoenfeld et al., 1989) is downregulated by 2 months (Nacimiento et al., 1993), suggesting that the connections are no longer malleable. However, our results indicate that the topography of CS tract connections can indeed be changed later in development, beyond the topographic refinement period. Topographic changes do not occur spontaneously over time (Friel and Martin, 2005), but require activity manipulation. We propose that tonic levels of activity in the initially active cortex maintain ipsilateral CS terminations that prevent the silenced CS terminations from establishing connections ventrally. In this way, the ipsilateral terminations are like place holders, preventing the silenced terminations from exerting their motor control signaling function. With the alternate blockade, ipsilateral terminations are withdrawn, opening synaptic space for the initially silenced CS projection.

\section{B Unilateral inactivation}
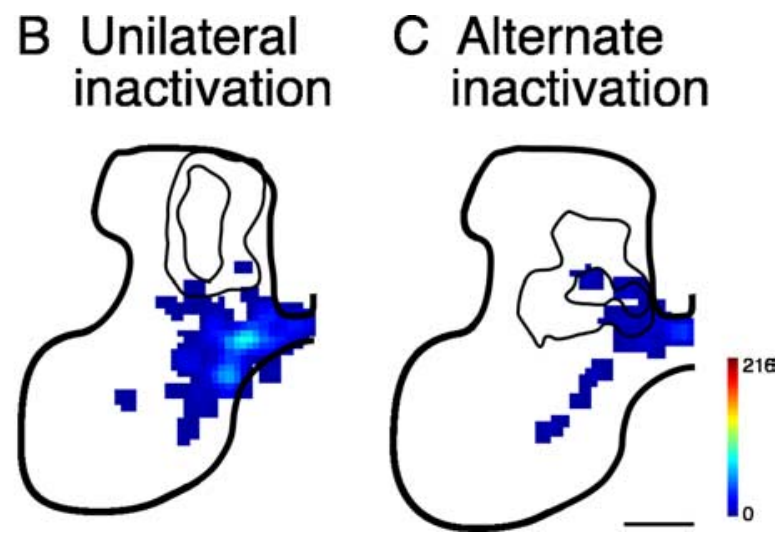

Figure 5. Alternate inactivation reduces ipsilateral $C S$ tract terminations. $A-C$, Averaged regional distribution of ipsilateral $C S$ Fions for controls $(n=4 ; \boldsymbol{A}$, replotted from Fig. $1 A)$, animals receiving unilateral inactivation only $(\boldsymbol{B} ; n=3)$ and aternate inactivation $(\boldsymbol{C} ; n=3)$. Conventions are similar to those in Figure $1 A$. We used either $\operatorname{LY}(n=2)$ or BDA $(n=1)$ to

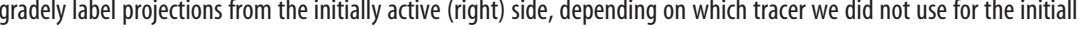
inactive side. The contours in $\boldsymbol{B}$ and $($ plot the region of maximal contralateral label (inner; $40 \%$; yellow) and an estimate of total

A

Figure 6. Alternate inactivation restores end point control during visually guided locomotion. $\boldsymbol{A}$, Forward distance of the forepaw contralateral to the first inactivation by week of representative cats after unilateral (filled circles) or alternate inactivation open circles). Forward distance remains higher than controls (dotted line, average, $n=4$ ) even 2 months after unilatera inactivation, forward distance returns to control levels 1 month after the second inactivation. Average control forward distance is as a dotted line and unilateral inactivation as a dashed line. $\boldsymbol{B}$, Plot showing the lack of recovery in animals with unilatera lines; $n=4$; data from a previous study for animals tested longer than 16 weeks) (Friel et al., 2007). After alternate inactivation (solid lines), all animals recovered in $<15$ weeks.

We have shown previously that inactivation of M1 after the early refinement period (e.g., PW7-PW11), in otherwise untreated animals, does not influence topography of CS tract axon terminations (Friel and Martin, 2005). However, in the present study, inactivation during the same late period redistributed the terminals of the initially inactivated side, from dorsal to more ventral laminas. This points to a protracted malleability of the previously inactivated axons. Early activity blockade during a critical period for CS circuit formation (PW5-PW7; Martin, 2005) appears to leave the silenced axons in a less mature state. The branching patterns and presynaptic bouton densities at PW15/16 match those of axons at age PW8 (Martin et al., 2004). Similar to immature axons, early activity blockade leaves axons susceptible to additional manipulations. Although the late inactivation did not affect the topography of contralateral terminations from the second inactivated side, it produced a partial withdrawal of the ipsilateral terminals from that side. This further suggests that the aberrant connections may be less stable than 
their normal counterparts. An important future direction will be to determine whether inactivation during the critical period results in a protracted period of GAP43 expression, indicating that the inactivated axons are susceptible to activity manipulations later in development. The increased malleability of the early inactivated CS axons could provide an important window for therapeutic intervention. After neonatal motor cortex lesions in rats, training during adulthood produced adaptive changes in the motor map of the lesioned hemisphere, indicating that the window for plasticity of the damaged CS axons extends into maturity (Williams et al., 2006).

\section{Bilateral CS interactions shape the function of the CS system}

Little is known about the mechanisms shaping development of the motor control functions of the CS system. Our findings suggest a novel view, in which the developing functions of the CS tract on one side of the spinal cord reflect the balanced contributions from the motor cortex on each side, rather than overwhelmingly from the contralateral side. Strong bilateral interactions clearly occur between PW5 and PW7, because M1 inactivation on one side leads to bilateral CS terminations from the other side (Martin et al., 1999; Friel and Martin, 2005). Our present findings show that, with the persistence of significant bilateral CS tract terminations, the alternate M1 inactivation between PW7 and PW11 has a strong effect on the ipsilateral side. Presumably, bilateral interactions normally persist during this late period of CS system development and into maturity because sparse ipsilateral terminations are present in the monkey and cat (Martin, 1996; Lacroix et al., 2004). In addition to a direct effect of ipsilateral CS axon terminations, indirect ipsilateral effects could be mediated at multiple levels of the CS system, including the corpus callosum, ipsilateral corticoreticular projections, and spinal commissural systems (Jankowska and Edgley, 2006).

Unlike the visual system, where bilateral interactions (e.g., binocular overlap in primary visual cortex) are required for context-independent depth perception, the limbs do not have such constraints; they can be controlled independently. Bilateral interactions at the spinal level during development may be important for establishing the circuitry for flexible bimanual coordination and for coordination between unilateral limb control and bilateral postural interactions. This study points to important interactions between the two sides that shape the structure and function of the CS system.

\section{Which CS circuit change is crucial for recovery?}

M1 inactivation produces defective contralateral endpoint control (Friel et al., 2007). This motor impairment could reflect any of the three key changes in CS connectivity produced by unilateral M1 activity restriction: (1) the lack of contralateral CS connections with appropriate ventral motor circuitry, (2) the presence of contralateral CS connections with inappropriate dorsal spinal circuitry, and (3) the presence of aberrant ipsilateral CS connections. Restoration of any or all of these circuit anomalies could contribute to normal endpoint control after alternate inactivation. It is not yet possible to dissociate the contributions of reducing ipsilateral terminations from redistributing contralateral terminations. Our results show that restitution of normal contralateral motor circuitry, rather than recruiting aberrant circuitry to work more effectively, is likely to be important in recovery.

The ipsilateral CS tract projection, a notable feature after activity blockade and in hemiplegic $\mathrm{CP}$, has been implicated in recovery of motor function after stroke in maturity (for a review of underlying circuitry, see Jankowska and Edgley, 2006). This role may be greater than that of the damaged contralateral projection after large strokes (Teasell et al., 2005). The laterality of the CS tract contributing to recovery may change during the poststroke period, with the ipsilateral projection more important soon after stroke, but less so 3-6 months later (Marshall et al., 2000). In contrast, some functional imaging studies report a negative correlation between ipsilateral activation and recovery, suggesting a maladaptive role of ipsilateral projections (Serrien et al., 2004). Although ipsilateral CS terminations may provide a substrate for supporting modest motor recovery after severe damage to the contralateral CS system, it appears that surviving contralateral terminations, when present, are most effective at driving motor recovery.

\section{Implications for hemiplegic cerebral palsy therapies}

Hemiplegic CP, a common movement disorder, is caused mostly by perinatal trauma to the developing CS system in one hemisphere. People with hemiplegic CP have reduced motor abilities contralateral to the damaged hemisphere and typically perform unimanual motor tasks solely with the unaffected side (Gordon et al., 2005; Charles et al., 2006). The organization of the CS system in patients with hemiplegic CP is characterized by the loss of motor responses evoked by transcranial magnetic stimulation (TMS) of the plegic side and emergence of bilateral responses from the unaffected side (Farmer et al., 1991; Carr et al., 1993; Eyre et al., 2001). This pattern is similar to the pattern of CS terminations that we have observed in the kitten after unilateral M1 inactivation (Martin et al., 1999). Babies who suffer neonatal strokes are at great risk for CP, yet surprisingly, the signs do not emerge for 1-2 years after birth (Bouza et al., 1994). Immediately after perinatal stroke, when signs are not present, TMS responses in babies that will later develop hemiplegic CP are similar to normal (Eyre et al., 2007). As these children grow older, TMS responses show an age-dependent decrease in the strength of responses evoked on the damaged side, along with a concurrent increase in the strength of bilateral responses evoked from the undamaged side. Indeed, young children with fast-conducting ipsilateral CS tract fibers have the most severe motor impairments (Eyre et al., 2007). The undamaged side appears to maintain ipsilateral projections, and these projections get stronger with age.

A therapeutic approach, constraint-induced movement therapy (CIMT), relies on increased use of the affected side in people with hemiplegic CP (for review, see Charles et al., 2006). CIMT has been shown to promote recovery of manual skill and motor maps after stroke (Wolf et al., 2006) or M1 damage in an animal model (Nudo et al., 1996). In the context of our findings, CIMT may restrict the developing functions of the unimpaired side by reducing neural activity to enhance the capabilities of the impaired side. CIMT improves generalized motor behavior in many children with hemiplegic CP (Gordon et al., 2005; Charles et al., 2006). Our findings provide a mechanism for understanding this efficacy: driving down activity of the undamaged CS system may enable the damaged CS system to reconnect more effectively with spinal motor circuits.

\section{References}

Alisky JM, Swink TD, Tolbert DL (1992) The postnatal spatial and temporal development of corticospinal projections in cats. Exp Brain Res $88: 265-276$.

Bouza H, Rutherford M, Acolet D, Pennock JM, Dubowitz LM (1994) Evolution of early hemiplegic signs in full-term infants with unilateral brain 
lesions in the neonatal period: a prospective study. Neuropediatrics 25:201-207.

Carr LJ, Harrison LM, Evans AL, Stephens JA (1993) Patterns of central motor reorganization in hemiplegic cerebral palsy. Brain 116:1223-1247.

Chakrabarty S, Martin JH (2000) Postnatal development of the motor representation in primary motor cortex. J Neurophysiol 84:2582-2594.

Chakrabarty S, Friel K, Martin JH (2007) Changes in M1 motor maps reflect plasticity of spinal terminations. Soc Neurosci Abstr 33:132.24.

Charles JR, Wolf SL, Schneider JA, Gordon AM (2006) Efficacy of a childfriendly form of constraint-induced movement therapy in hemiplegic cerebral palsy: a randomized control trial. Dev Med Child Neurol 48:635-642.

Eyre JA, Taylor JP, Villagra F, Smith M, Miller S (2001) Evidence of activitydependent withdrawal of corticospinal projections during human development. Neurology 57:1543-1554.

Eyre JA, Smith M, Dabydeen L, Clowry GJ, Patacchi E, Battini R, Guzzetta A, Cioni G (2007) Is hemiplegic cerebral palsy equivalent to amblyopia of the corticospinal system? Ann Neurol, in press.

Farmer SF, Harrison LM, Ingram DA, Stephens JA (1991) Plasticity of central motor pathways in children with hemiplegic cerebral palsy. Neurology 41:1505-1510.

Friel KM, Martin JH (2005) Role of sensory-motor cortex activity in postnatal development of corticospinal axon terminals in the cat. J Comp Neurol 485:43-56.

Friel KM, Drew T, Martin JH (2007) Differential activity-dependent development of corticospinal control of movement and final limb position during visually-guided locomotion. J Neurophysiol 97:3396-3406.

Gordon AM, Charles J, Wolf SL (2005) Methods of constraint-induced movement therapy for children with hemiplegic cerebral palsy: development of a child-friendly intervention for improving upper-extremity function. Arch Phys Med Rehabil 86:837-844.

Jankowska E, Edgley SA (2006) How can corticospinal tract neurons contribute to ipsilateral movements? A question with implications for recovery of motor functions. Neuroscientist 12:67-79.

Lacroix S, Havton LA, McKay H, Yang H, Brant A, Roberts J, Tuszynski MH (2004) Bilateral corticospinal projections arise from each motor cortex in the macaque monkey: a quantitative study. J Comp Neurol 473:147-161.

Li Q, Martin JH (2001) Postnatal development of corticospinal axon terminal morphology in the cat. J Comp Neurol 435:127-141.

Li Q, Martin JH (2002) Postnatal development of connectional specificity of corticospinal terminals in the cat. J Comp Neurol 447:57-71.

Marshall RS, Perera GM, Lazar RM, Krakauer JW, Constantine RC, DeLaPaz RL (2000) Evolution of cortical activation during recovery from corticospinal tract infarction. Stroke 31:656-661.

Martin JH (1996) Differential spinal projections from the forelimb areas of the rostral and caudal subregions of primary motor cortex in the cat. Exp Brain Res 108:191-205.

Martin JH (2005) The corticospinal system: from development to motor control. Neuroscientist 11:161-173.

Martin JH, Lee S (1999) Activity-dependent competition between developing corticospinal terminations. NeuroReport 10:2277-2282.

Martin JH, Kably B, Hacking A (1999) Activity-dependent development of cortical axon terminations in the spinal cord and brain stem. Exp Brain Res 125:184-199.

Martin JH, Hacking A, Donarummo L (2000) Impairments in prehension produced by early postnatal sensorimotor cortex activity blockade. J Neurophysiol 83:895-906.

Martin JH, Choy M, Pullman S, Meng Z (2004) Corticospinal development depends on experience. J Neurosci 24:2122-2132.

Martin P, Bateson P (1985) The ontogeny of locomotor play behavior in the domestic cat. Animal Behav 15:59-103.

Meng Z, Martin JH (2003) Postnatal development of corticospinal synaptic actions. J Neurophysol 90:683-692.

Meng Z, Li Q, Martin JH (2004) The transition from development to motor control function in the corticospinal system. J Neurosci 24:605-614.

Nacimiento W, Topper R, Fischer A, Oestreicher AB, Nacimiento AC, Gispen WH, Noth J, Kreutzberg GW (1993) Immunocytochemistry of B-50 (GAP-43) in the spinal cord and in dorsal root ganglia of the adult cat. J Neurocytol 22:413-424.

Nudo RJ, Wise BM, SiFuentes F, Milliken GW (1996) Neural substrates for the effects of rehabilitative training on motor recovery after ischemic infarct. Science 272:1791-1794.

Schoenfeld TA, McKerracher L, Obar R, Vallee RB (1989) MAP 1A and 1B are structurally related microtubule associated proteins with distinct developmental patterns in the CNS. J Neurosci 9:1712-1730.

Serrien DJ, Strens LH, Cassidy MJ, Thompson AJ, Brown P (2004) Functional significance of the ipsilateral hemisphere during movement of the affected hand after stroke. Exp Neurol 190:425-432.

Teasell R, Bayona NA, Bitensky J (2005) Plasticity and reorganization of the brain post stroke. Top Stroke Rehabil 12:11-26.

Theriault E, Tatton WG (1989) Postnatal redistribution of pericruciate motor cortical projections within the kitten spinal cord. Dev Brain Res 45:219-237.

Williams PT, Gharbawie OA, Kolb B, Kleim JA (2006) Experiencedependent amelioration of motor impairments in adulthood following neonatal medial frontal cortex injury in rats is accompanied by motor map expansion. Neuroscience 141:1315-1326.

Wolf SL, Winstein CJ, Miller JP, Taub E, Uswatte G, Morris D, Giuliani C, Light KE, Nichols-Larsen D (2006) Effect of constraint-induced movement therapy on upper extremity function 3 to 9 months after stroke: the EXCITE randomized clinical trial. JAMA 296:2095-2104. 